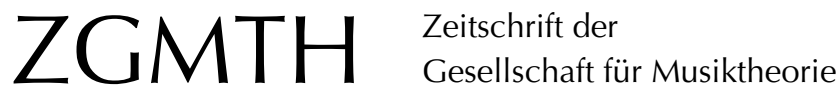

Roth, Markus (2010): Les Ombres Errantes. Vier Sichtweisen auf Satztechnik und Kombinatorik bei François Couperin. ZGMTH 7/2, 119-133.

https://doi.org/10.31751/514

(C) 2010 Markus Roth

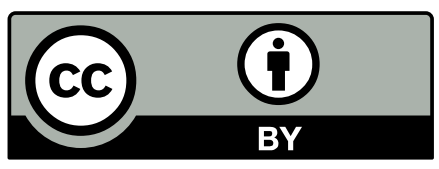

Dieser Text erscheint im Open Access und ist lizenziert unter einer Creative Commons Namensnennung 4.0 International Lizenz.

This is an open access article licensed under a Creative Commons Attribution 4.0 International License.

veröffentlicht / first published: 05/07/2010

zuletzt geändert / last updated: 02/07/2010 


\section{Les Ombres Errantes}

\section{Vier Sichtweisen auf Satztechnik und Kombinatorik bei François Couperin}

Markus Roth

Die vorliegende Studie versammelt vier analytische Sichtweisen auf Couperins Pièce Les Ombres Errantes: eine historisch orientierte, eine modellbezogene, eine schenkerianische und eine motivische. Die Widersprüche, die durch die Gegenüberstellung verschiedener analytischer Ansätze entstehen, werfen Fragen auf, die auf die Rätselhaftigkeit des Stückes zurückverweisen.

Die selbstverständliche Konkurrenz zwischen verschiedenen Theorien und Analysesystemen ist ein hervorstechendes Merkmal der Vielschichtigkeit, die den gegenwärtigen musiktheoretischen Diskurs bestimmt. ${ }^{1}$ Analyse betreiben, bedeutet vor diesem Hintergrund keine geringe Herausforderung - eine Herausforderung, der am ehesten mit einem reflektierten Methodenpluralismus zu begegnen ist: Jeder Wechsel des Blickwinkels schärft den Blick für bislang verborgene oder vernachlässigte Details; das Ineinandergreifen verschiedener Sichtweisen gewährleistet Transparenz und Tiefenschärfe. Der Einbezug eher spekulativer Ansätze wird möglich, ohne sich systemimmanenten Deutungszwängen auszuliefern. Ein solcher Methodenpluralismus lässt sich als Versuch verstehen, ein Werk (in Anlehnung an einen verführerischen Gedanken von Carl Dahlhaus) mit möglichst praktikablen `Gerüsten` zu umstellen - mit Gerüsten, die man wieder abbauen kann oder gar abbauen sollte, wenn sie ihren Dienst getan haben. ${ }^{2}$

In der analytischen Praxis jedoch wirft dies einige grundsätzliche Schwierigkeiten auf. Denn das analytische Instrumentarium, das es von Fall zu Fall (von Stück zu Stück) neu zu konfigurieren und an den Besonderheiten des jeweiligen Gegenstandes auszurichten gilt, ist keineswegs sneutral : Zur Auswahl stehen nicht bloße >Methoden`, sondern Systeme mit bedeutsamer Vorgeschichte. Daher bedingt das Nebeneinander verschiedener Sichtweisen auch ein (möglicherweise widersprüchliches) Nebeneinander von Begrifflichkeiten, in denen sich bestimmte historische Denkweisen verfestigt haben. ${ }^{3}$ Zudem

1 Einen Überblick über den deutschsprachigen Diskurs bietet der Band Systeme der Musiktheorie (Kühn/Leigh 2009).

2 Siehe Dahlhaus 1984, 76, sowie ergänzend Schwab-Felisch 2004, 349-353. Die Forderung, Theorien als bloße ‘Gerüste` zu verwenden, steht bei Dahlhaus ursprünglich im Kontext einer grundsätzlichen Kritik an der Schichtenlehre Heinrich Schenkers (vgl. Schwab-Felisch 2004, S. 352).

3 Zur Anwendung verschiedener historischer Analysemethoden auf die ersten sechs Takte des zweiten Satzes der Waldstein-Sonate siehe Jans 1997. 
sind die wenigsten Theorien - seien es ältere wie die Schichtenlehre Heinrich Schenkers, seien es jüngere oder jüngste wie etwa die `Neo-Riemannian-Theory - mit anderen kompatibel.

Vor dem skizzierten Hintergrund versammelt der folgende Text vier Sichtweisen auf Couperins Pièce Les Ombres Errantes aus dem Quatrième Livre de pièces de Clavecin, das 1730 in Paris im Druck erschien. Die nachstehenden Analysen versuchen eine möglichst umfassende Rekonstruktion der kompositorischen Denkweisen, die das Stück so geraten ließen, wie es vorliegt; darüber hinaus soll reflektiert werden, welche Konsequenzen ein Wechsel der analytischen Perspektive für die musikalische Wahrnehmung hat. Allerdings erwies es sich als schwierig, die Vielzahl angerissener Aspekte und Einzelbeobachtungen überhaupt in eine lesbare Form zu bringen und den komplexen Erkenntnisprozess nachvollziehbar zu machen, der durch die Gegenüberstellung und Durchdringung verschiedener Sichtweisen befördert wird. Zu den `Spielregeln` des Textes gehört daher die Option, einmal formulierte Erkenntnisse durch Beobachtungen an anderer Stelle zu relativieren. Statt wie üblich vom Ganzen ins Detail zu gehen, arbeiten die folgenden Abschnitte I-IV zunächst bestimmte Einzelaspekte heraus, so dass ein 'Mosaikı aus Beobachtungen entsteht. Während Abschnitt I - ausgehend von einigen stilistischen Besonderheiten des Cembalo-Satzes - eine primär historische Perspektive verfolgt, greifen die beiden nachfolgenden Analyse-Fragmente zwei aktuelle methodische Ansätze auf: Abschnitt II reflektiert Vorzüge und Grenzen einer Analyse nach Klangfortschreitungs-Modellen, Abschnitt III diskutiert Aspekte einer StimmführungsAnalyse nach Heinrich Schenker. Abschnitt IV schließlich versucht, Couperin im Geiste Schönbergs zu betrachten.

\section{Vier Perspektiven}

François Couperins Quatrième Livre de pièces de Clavecin enthält insgesamt 45 Stücke in sieben `ordres`; Les Ombres Errantes beschließt `ordre`25. ${ }^{4} \mathrm{Im}$ Folgenden wird dem zyklischen Kontext des Stückes keine nähere Beachtung geschenkt; ohnehin scheint `ordre ‘ beim späten Couperin zunächst nichts anderes zu bedeuten als eine lockere Zusammenfassung von einzelnen `Charakterstücken` mit gemeinsamer Tonart. ${ }^{5}$ Allerdings sah sich Couperin veranlasst, im Vorwort zur Erstausgabe des »Quatrième Livre» die

4 Les Ombres Errantes liegt in folgenden Editionen vor: 1.) François Couperin, Pièces de Clavecin, Quatrième livre, Edition par Kenneth Gilbert (= Le Pupitre, Bd. 24), Paris 1972; 2.) OEuvres complètes de François Couperin: Pièces de Clavecin 4, publiées par M. Cauchie et revues d'après les sources par Kenneth Gilbert, Monaco 1982. Die Analyse beruht auf dem Notentext der letztgenannten Ausgabe.

5 Arnfried Edler sieht in Couperins Vorliebe für eher lockere zyklische Ordnungen eine »Verweigerung großer systematisch-spekulativer Zusammenhänge [...] [in] der literarischen Tradition Montaignes und Pascals, die in der fragmentarischen Struktur der Literatur einen angemessenen Ausdruck für die Begrenzung und Dissoziation menschlicher Erkenntnis durch die Bedingungen der Subjektivität erblickten.« (1997, 208) Der Pianist Alexandre Tharaud (tic toc choc. Alexandre Tharaud joue Couperin, siehe Anm. 15) leitet aus diesem Sachverhalt die Forderung ab, die von Couperin vorgeschlagenen Ordnungen bewusst aufzusprengen und Stücke aus verschiedenen Zusammenhängen auf neue Weise zu kombinieren. 
ungewöhnliche erweiterte Tonartenfolge des `ordre` 25 zu erläutern, und gab zudem mit dem Hinweis auf zwei angeblich verlorengegangene Stücke erste Rätsel auf. ${ }^{6}$ In populären Darstellungen gilt Les Ombres Errantes, umrankt vom Nimbus des Spätwerks, häufig als reinstes Konzentrat des Couperin-Stils ${ }^{7}$, wenn nicht gar als Essenz der französischen Musik schlechthin.

Die auffälligste Eigenart des Stückes ist die Regelmäßigkeit seines Phrasenbaus. Jede Phrase umfasst acht Viertel, jede Reprise sieben Phrasen, die durch Kadenzen verschiedener Stärke deutlich voneinander getrennt werden; fasst man größere Einheiten zusammen, so ergibt sich in beiden Reprisen eine Gliederung von 4+4+4+2 Takten. (Der letzte Zweier wirkt wie ein Anhang und dient lediglich zur Bekräftigung der Kadenz. ${ }^{8}$ ) Ungewöhnlich die metrische Notation mit einem dreizeitigen Auftakt, der durch die Dauer der Schlussklänge von nur einem Viertel aufgefangen wird. Sieht man auf die Verzierungszeichen und die später folgenden Appoggiaturen, so ergeben sich auftaktige Zweiereinheiten (Beispiel 1). ${ }^{9}$

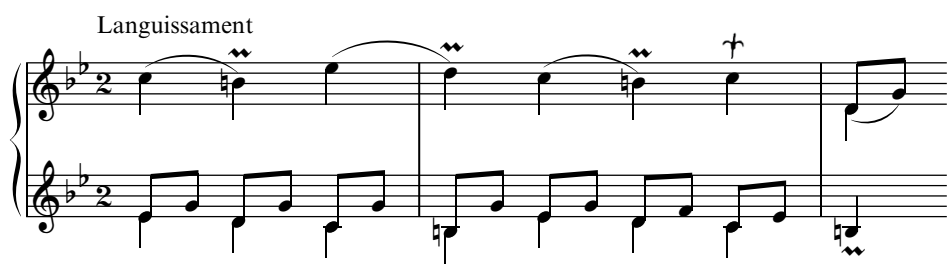

Beispiel 1: Les Ombres Errantes, Beginn

Mit der Starrheit des Phrasenbaus korrespondiert die Gleichförmigkeit der Bewegung; zu den in Vierteln fortschreitenden Außenstimmen, die ein Gerüst aus Terzen (Dezimen) und Sexten bilden, treten eine, seltener zwei nachschlagende synkopierende Mittelstimmen. Die Überschrift »Languissamment« (`ermattend`) impliziert ein `recht langsames Tempo.${ }^{10}$ Wenngleich der Notentext eine gewisse Gleichförmigkeit suggeriert, wird Couperin sicherlich mit einem flexiblen sinegalen، Spiel des Interpreten gerechnet haben; dennoch ist die Beschränkung unübersehbar, die der Komponist sich selbst auferlegt: Über weite Strecken wirkt das Satzbild des Stückes wie ein Skelett, dem die Ornamentation fehlt.

6 Vgl. das Vorwort zu Le Pupitre 24, X-XI. - Die Tonartfolge von `ordre 25 lautet Es-Dur (La Visionaire), C-Dur (La Misterieuse), c-Moll (La Monflambert), C-Dur (La Muse Victorieuse), c-Moll (Les Ombres Errantes).

7 »[...] I'une des plus fluides, I'une des plus couperiniennes de toutes les pièces, I'une de celles où semble se concentrer tout ce qui fait la caractéristique de son style, et sa poésie." (Beaussant 1980, 489)

8 Der harmonische Grundriss mit förmlichen Ausweichungen in die Tonart der dritten und fünften Stufe entspricht den Konventionen eines barocken Suitensatzes.

9 Die Taktzählung im Folgenden in Entsprechung zur Cauchie-Edition (siehe Anm. 4).

10 Siehe Miehling 2003, 225; man vgl. Couperins Rondeau La Muse Plantine (Livre III, ordre 19). 


\section{Schattenwürfe, Verschleierung und ıstyle brisés}

Das Motiv `Unstete Schatten lässt fortwährende Bewegung und darüber hinaus Unbestimmtheit, Unschärfe, Rätselhaftigkeit assoziieren; im Lichte der Titel-Metapher gleicht Les Ombres Errantes einer Studie über gehäufte Vorhaltsbildungen und zzerfließende` Harmonien, die der Versinnbildlichung von Schattenwürfen dienen. Die ästhetischen und satztechnischen Eigenarten des Stückes stehen in der Tradition des sogenannten sstyle brisé‘: Ausgangspunkt der Entstehung einer eigenständigen französischen Clavecin-Musik im 17. Jahrhundert war der Versuch einer Übertragung bzw. Nachahmung bestimmter idiomatischer Merkmale des Lautenstils.

Merkmale des sstyle brisé sind eine weitgehende Freistimmigkeit (mit frei hinzutretenden und wieder verschwindenden Stimmen), eine eher lockere Stimmführung, die Imitation lautenartiger Figuren und Akkordbrechungen sowie die Übernahme einer Vielzahl von lautentypischen Ornamenten; durch die klangliche Auffächerung des zugrundeliegenden Generalbass-Gerüsts entsteht ein gebrochener, in sich sverschobener Satz. ${ }^{11}$ Um einen solchen handelt es sich - ungeachtet der Einfachheit des Satzbildes - auch im vorliegenden Stück. Zwei Belegstellen mögen die satztechnischen Komplikationen verdeutlichen, die sich unter diesen Vorzeichen ergeben.
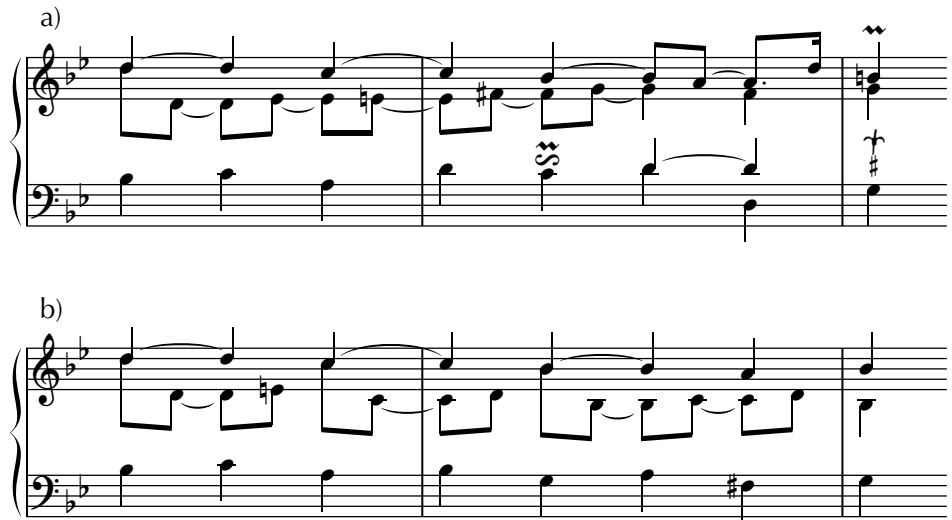

Beispiel 2: a) Les Ombres Errantes, T. 18-20; b) Integration in einen regelmäßigen Sequenzgang

Der doppelte Nonenvorhalt auf dem dritten Viertel von Takt 18 ist - zumal in einem dreistimmigen Kontext - sicherlich eine Rarität. Ermöglicht wird er durch den Umstand, dass der gebundenen None zwei Wege der Auflösung offen stehen, nämlich sowohl der Sekundschritt nach unten als auch nach oben. ${ }^{12}$ Der Mechanismus ließe sich, wie Beispiel $2 \mathrm{~b}$ belegt, unschwer in einen regelmäßigen Sequenzgang integrieren. Im zitierten Ausschnitt führt die synkopische Führung der Mittelstimme mit ihren »rückenden No-

11 Manche Effekte der Verunklarung des Klangs, z. B. durch Resonanzwirkungen auf weiterklingenden leeren Saiten, lassen sich auf der Laute gar nicht vermeiden. 


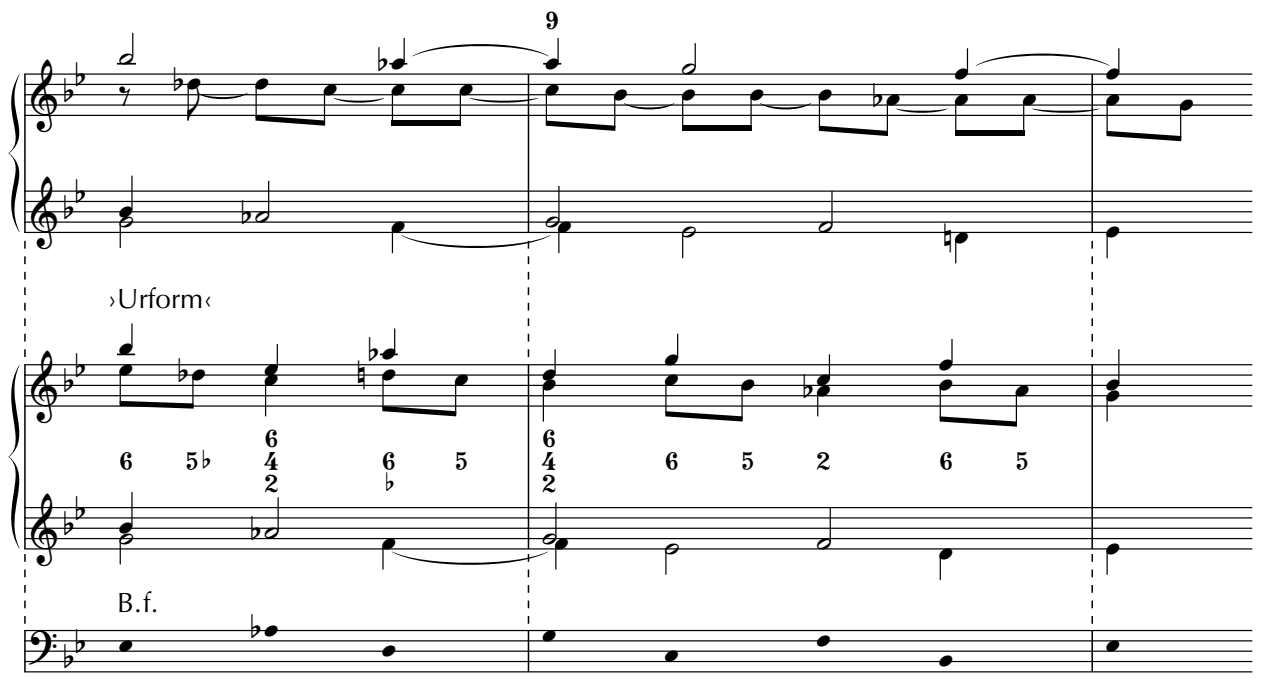

Beispiel 3: Les Ombres Errantes, T. 8-10, Quintfall-Mechanismus mit Basse Fondamentale

ten« (Kirnberger) zu einer sungleichzeitigen`, um ein Achtel verschobenen Auflösung der doppelten Dissonanz; die aufwärts geführte None im Alt wird zum Ausgangspunkt des aufsteigenden Quartganges $d^{1}-e s^{1}-e^{1}-f i s^{1}-g^{1}$, so dass Alt und Diskant einen sich schließenden `Fächer bilden. Die Wirkung der Stelle beruht vor allem auf der Führung der Mittelstimme: Man beachte die übermäßige Quinte e (T. 18, letztes Achtel) und das Aufeinandertreffen von $c^{1}$, fis ${ }^{1}$, und $b^{1}$ auf der zweiten Zählzeit von Takt $19 .{ }^{13}$ Die fraglichen Takte enthalten mehrere Dissonanztypen (in historischen Begriffen: 'syncopatio‘, sretardatio`, stransitus`) in verschiedenen Werten; ihre Häufung verweist auf einen besonderen Affekt.

In den Takten 9-10 (Beispiel 3) hingegen beruht der Effekt des `Zerfließens` der Harmonie auf einer Häufung von Vorhaltsbildungen im durchweg vierstimmigen Satz. Als Agensstimme fungiert (wie in den entsprechenden Takten zu Beginn der zweiten Reprise) allein der Tenor; in Halben fortschreitend, zieht er gleichsam einen Schweif von Dissonanzen nach sich, deren ungleichzeitige Auflösung die kalkulierte Wirkung der Passage - die Versinnbildlichung von Unschärfe - noch verstärkt. Letztlich lassen sich die betreffenden Takte, wie Beispiel 3 veranschaulicht, auf ein Quintfall-Modell zurückführen, dessen Besonderheit darin besteht, dass jeder zweite Ton der zugrundeliegenden

12 Mattheson verweist in diesem Zusammenhang auf den Umstand, „daß man ehmahls mit der Secunde eben also zu Wercke gegangen sey, welche auch, bey etlichen der gründlichsten Contrapunctisten [als Johann Theile und Zarlin ein Paar gültige Zeugen] [...] sowohl durch den Einklang, als durch die Tertz, geborgen wird.« $(1739,326)$

13 Markiert wird die zweite Zählzeit zudem durch die Verzierung (`tremblement et doublés) in der Unterstimme. - Parallele Septimen, wie hier in Takt 19 in den Außenstimmen, trifft man bei Couperin in Klauselnähe häufiger an, z. B. im Rondeau La Favorite (ordre 3), Refrain, Takt 4 bzw. 8. 
Quinten-Reihe real gar nicht erklingt. Merkwürdig die nachschlagenden Oktaven zwischen Tenor und Diskant, deren Duldung sich vermutlich den besonderen Bedingungen des sstyle brisé ‘ verdankt; über den angenommenen Fundament-Tönen as, $g$ und $f$ ergeben sich Nonenakkorde, wobei durch die Quartvorhalte in der Altstimme zusätzliche Spannungsmomente entstehen. ${ }^{14}$

Die Grafik verdeutlicht, dass diese Faktur - und dementsprechend auch der vverschleierte Quintfallı in den Takten 14-16 - als individuelle Ausprägung eines gängigen Musters erklärt werden kann. Eine ähnliche Häufung von Ligaturen über einem gedachten Quintfall-Fundament findet sich bei Couperin in einem über zehn Jahre älteren Prélude (Beispiel 4), hier noch mit einer zusätzlichen Stimme und in einem dunkleren Register, das die charakteristischen Verwischungseffekte noch verstärkt.

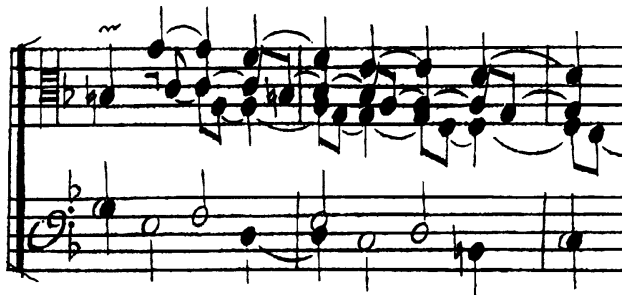

Beispiel 4: François Couperin, L'Art de toucher le clavecin (1717), Quatrième Prélude, T. 9-11 (vgl. Les Ombres Errantes, T. 14-16)

Die von Couperin imaginierten Verwischungseffekte kommen bei einer Wiedergabe auf einem modernen Instrument natürlich viel stärker zur Geltung als auf einem Cembalo. ${ }^{15}$ Couperins Raffinesse, Momente wie Unbestimmtheit und Unschärfe musikalisch zu versinnbildlichen, weist bereits auf bestimmte Préludes von Debussy voraus (ich denke hier vor allem an die Nr. 1, Brouillards, aus dem Deuxième Livre); und setzt man an einigen ausgewählten Stellen vertikale Schnitte an - z. B. auf der zweiten Zählzeit von Takt 14 - so stößt man auf verblüffend `moderne`, nahezu clusterartige Zusammenklänge (hier: es-f-g $\left.-a s^{1}\right)$.

\section{Stück-Werk}

Les Ombres Errantes ist eine reiche Fundgrube für satztechnische Modelle. Über die Möglichkeiten und Vorzüge einer an Satzmodellen orientierten Analyse ist in jüngster Zeit viel diskutiert worden. ${ }^{16}$ Wer über Satz- oder besser über »KlangfortschreitungsModelle« spricht, muss zwischen ihren grundlegenden strukturellen Merkmalen, As-

14 Zum »Septimennonenaccord mit dazu genommener Quarte statt Terz« vgl. Bach 1762, 167; an anderer Stelle findet sich auch ein Beispiel zur Folge von Nonenvorhalt und Quintsextakkord über einem Quintfall-Fundament (ebd., 158).

15 In jüngster Zeit hat Alexandre Tharaud demonstriert, wie gut Couperin auch auf einem modernen Flügel klingen kann, vgl. Tic toc choc. Alexandre Tharaud joue Couperin, harmonia mundi 901956 (2007). Ich empfehle einen Vergleich mit der auf dem Cembalo eingespielten Interpretation von Mitzi Meyerson (Glossa 921802 [2004]).

16 Zum aktuellen Stand der Diskussion siehe u. a. die Ausgaben 4/1-2 und 4/3 der ZGMTH. 
pekten ihrer stil- und zeitgebundenen Auskomponierung und der ihnen anhaftenden `Bedeutung` unterscheiden. (Von semantischen Konnotationen eines Modells lässt sich sinnvoll nur im Rekurs auf dessen jeweiligen historischen Kontext sprechen, und naturgemäß ist dieser Punkt der heikelste.) Ein an Modellen orientiertes analytisches Hören verändert die musikalische Wahrnehmung, es schärft die Wahrnehmung für Bruchstellen im Gefüge der Tonalität. ${ }^{17}$ So droht Les Ombres Errantes, je länger das Stück dauert, beim Hören in Einzelteile zu zerfallen: Indem sich Muster an Muster aneinanderreiht, scheint es, als werde einfach an Satzmodellen sentlang komponierts, ein Eindruck, der durch die beschriebene Gleichförmigkeit des Phrasenbaus noch verstärkt wird. Insofern kann Couperins Pièce, um mit Martin Ebyl zu sprechen, als »vernetztes Stückwerk» in ganz besonderem Sinne gelten. ${ }^{18}$

Bei den in Beispiel 5 zitierten Ausschnitten handelt es sich um charakteristische Ausprägungen gängiger Satzmodelle: So beruht der Außenstimmensatz der Takte 4-6 auf einem (kanonischen) Gegenschritt-Modell ${ }^{19}$, die Takte 22-24 repräsentieren einen Quintstieg-Mechanismus ${ }^{20}$ als Folge zweier Halb-Kadenzen in steigender Progression. Ihren Reiz gewinnen beide Stellen durch die Führung der Mittelstimme(n): Im ersten Ausschnitt wird der Außenstimmenkanon der Takte 4-6 auf überraschende Weise mit einem aufwärts strebenden chromatischen Gang im Alt kombiniert, im zweiten bürgen Quartvorhalte und Dur-Moll-Wechsel in derselben Stimme für zusätzliche Spannungsmomente. Wie die gestrichelten Klammern verdeutlichen, weist die Führung der Altstimme hier zudem auf den Beginn des Stückes, konkret auf die ersten vier Töne im Diskant zurück. Die Raffinesse des Klaviersatzes beruht zu einem großen Teil auf chromatischen Schärfungen der Mittelstimme(n), die die zugrundeliegenden Satzmodelle in hohem Maße individualisieren. ${ }^{21}$ Vor allem die Entsprechung der Gerüsttöne im Diskant lässt es plausibel erscheinen, beide Stellen hörend aufeinander zu beziehen: Die strukturelle Verwandtschaft zwischen den zugrundeliegenden Satzmodellen ermöglicht es Couperin, das charakteristische `Terz-Quart-Zickzackı des Gegenschritt-Modells fast beiläufig in die Quintstieg-Sequenz zu integrieren.22 Dem kanonischen Außenstimmengerüst des Gegenschritt-Modells entspricht vor diesem Hintergrund das latent kanonische

17 Diese Sensibilität mag sich auch Erfahrungen mit Neuer Musik verdanken, in der das sbruchlose Ganze bekanntlich eine höchst problematische Kategorie geworden ist.

18 Siehe Ebyl 2005, 54-66. - „Der Begriff des Stückwerks bezeichnet etwas aus Komponenten zusammengefügtes; in diesem Sinn ist er ein Äquivalent zu `Komposition`. Tonale Musik ist ein Ganzes aus heterogenen Teilen.« (Ebd., 59)

19 Zur Herkunft und Bedeutung von Gegenschritt-Modellen siehe ausführlich Froebe 2007 (zum Terminus ,Gegenschritt ebd. 15, Anm. 8). Verschiedene Formen sequenzieller Kanonmodelle spielten in der Praxis der mehrstimmigen Vokalimprovisation im 16. und 17. Jahrhundert eine wichtige Rolle.

20 Zur Grundform des Quintstieg-Modells siehe die Lehrbeispiele 6 und 10 aus Händels Aufgaben zur Generalbasslehre (Händel 1978, 23 und 25). Als Muster kann der Beginn des zweiten Satzes aus Händels Orgelkonzert op. 4 Nr. 4 gelten.

21 Philippe Beaussant spricht mit Blick auf Les Ombres Errantes metaphorisch von einem "zweiten Diskurs« der Mittelstimme(n) (1980, 489-490).

22 Im Auftakt zu Takt 11 bringt die Oberstimme ein ähnliches `Terz-Quart-Zickzackı in diminuierter Form. 
Verhältnis der Oberstimmen des Quintstieg-Modells, das im vorliegenden Fall allerdings weitgehend verwischt wird - zugunsten einer Profilierung der synkopierten und chromatisierten Altstimme.
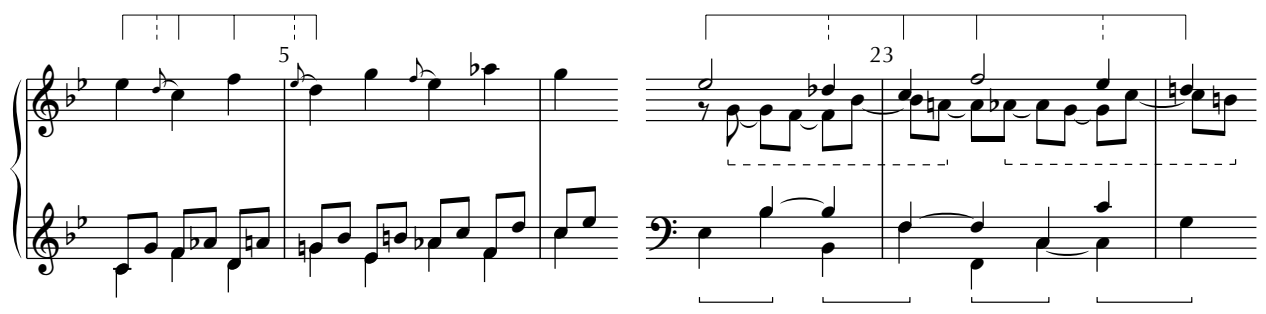

Beispiel 5: Gegenschritt und Quintstieg

Schließlich kann eine an Modellen orientierte Analyse Aufschluss über die besondere Art und Weise geben, wie Musik zusammengefügt, ıkomponiert ist; hilfreich kann in diesem Zusammenhang auch der Versuch sein, das betreffende Stück aus seinen einzelnen Teilen bzw. Bruchstücken auf ganz andere Weise zusammenzusetzen. Für die schlaglichtartige Charakterisierung dieser einzelnen Teile wurden in der nachstehenden Grafik, soweit möglich, gängige Modell-Bezeichnungen benutzt; ${ }^{23}$ saufgehoben` sind die einzelnen (steigenden oder fallenden) Modelle in übergreifenden melodischen Bewegungen, von denen Abschnitt III ausführlicher handelt. Die Übersicht veranschaulicht zudem das Netz aus harmonischen Zäsuren verschiedener Stärke und Prägnanz, welches das Stück überspannt. ${ }^{24}$ Hinzuzufügen bleibt, dass einige Details der Kadenz-Hierarchie gegen die Starrheit der angenommenen Gliederung $(4+4+4+2)$ arbeiten: Man beachte den betont schwachen Abschluss in Takt 8 sowie den starken, durch die picardische Terz $h^{1}$ markierten Schnitt in Takt 20 (inmitten des zweiten Vierers der zweiten Reprise).

Stark geprägt wird Les Ombres Errantes darüber hinaus durch den besonderen Gebrauch kontrastierender Lagen. Bis Takt 12 herrscht ein betont helles, transparentes Klangbild vor unter weitgehender Aussparung der Bassregion; umso überraschender wirkt der 'Lagen-Sturz im zweitaktigen Anhang der ersten Reprise (T. 12-14 und entsprechend T. 26-28), der die nahende zentrale Zäsur gleichsam mit instrumentatorischen Mitteln markiert.

23 »Die schlichte Frage, was ein Satzmodell ausmacht, ist freilich nicht leicht zu beantworten. Sollen historisch so disparate Gebilde wie die ı7-6-Progression` und der `Ruggierobass`, die `Initialkadenz und die sTeufelsmühle، allesamt als Satzmodelle gelten, verbietet sich eine historisch-genetische Bestimmung. Ein systematischer Modellbegriff aber, der sich dem Vorwurf der Willkür nicht aussetzen möchte, steht vor der Schwierigkeit, dass die Modellbegriffe, auf die er sich stützen könnte, nicht minder heterogen sind denn die Modelle selbst.« (Schwab-Felisch/Fuß 2007, 9)

24 Weiteren smotivischen Verbindungen zwischen den einzelnen Teilen bzw. Phrasen wird in Abschnitt IV nachgegangen. 


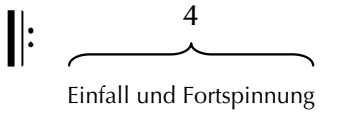

c.p.

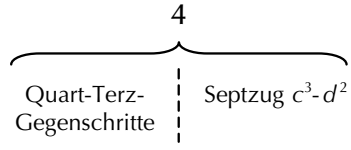

C.i.

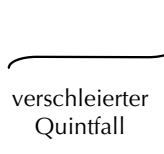

C.p.r.
(III)
4

Einleitung der Anhang Kadenz

c.p.

c.p.

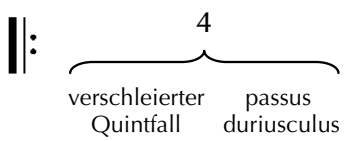

Quintfall duriusculus

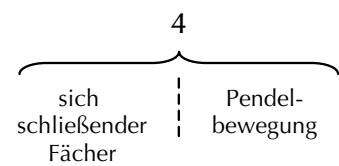

Fächer

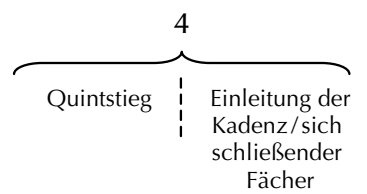

2

Anhang

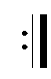

C.i.
(I)

C.p.
(III)

C.i.
(V)

c.p.
(V)

c.p.

(I)

c.p.

c.p.
(I)

Abbildung 1: Les Ombres Errantes, Formplan; Abkürzungen: c. p. = ‘cadence parfaite`, c. i = ‘cadence imparfaite`, c. p. r. = scadence parfaite renversée $\iota^{25}$

\section{Mit Schenkers Augen}

Da die Oberstimme von Les Ombres Errantes im Prinzip aus lauter fallenden ¿Zügen besteht, drängt sich eine Analyse im Sinne Heinrich Schenkers geradezu auf: Verbindet man die lokalen Spitzentöne der immer wieder neu ansetzenden abgleitenden Skalenbewegungen, so lässt sich unschwer ein übergreifender melodischer Zusammenhang konstruieren, der dem Prinzip des sfließenden Gesangs` gehorcht.

Es ist hier nicht der Ort, eine erschöpfende Schichten-Analyse auszuführen oder gar auf immanente Probleme der Schenker-Lehre einzugehen. Allerdings springen einige Details ins Auge, die die bislang formulierten Beobachtungen auf interessante Weise ergänzen. Schon ein flüchtiger Blick auf die folgende Mittelgrundskizze (Beispiel 6) belegt die besondere Bedeutung von Quintzügen für das gesamte Stück; vor diesem Hintergrund lassen sich die Takte 24-26 als gedrängte Zusammenfassung des Ganzen interpretieren.

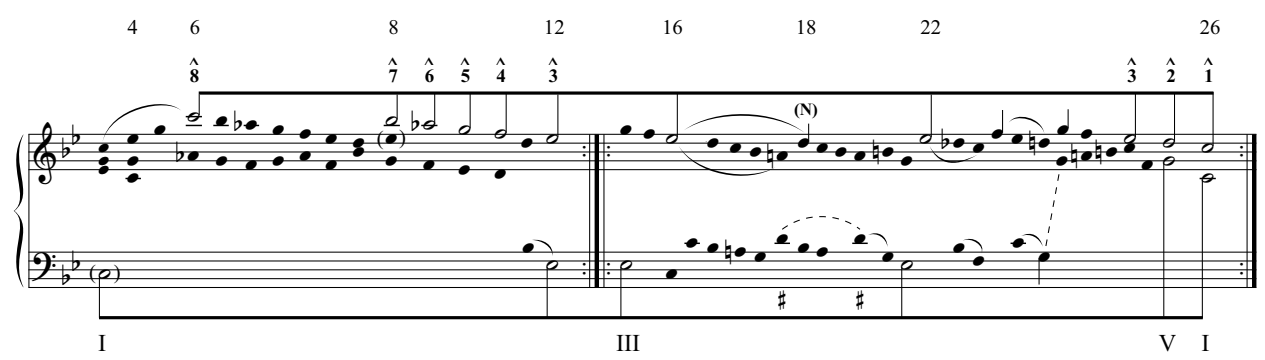

Beispiel 6: Mit Schenkers Augen (nach David Smyth ${ }^{26}$ )

25 Kadenzbezeichnungen nach Rameaus Traité von 1722, ungeachtet ihrer Unstimmigkeiten, die vor allem die `cadence imparfaite` (bzw. `cadence irrégulière $\triangleleft$ ) betreffen.

26 Die analytische Reduktion wurde mit marginalen Änderungen und Ergänzungen von Smyth übernommen (1999, 122). 
Nimmt man ferner das durch einen Quartsprung erreichte $c^{3}$ in Takt 6 als Kopfton eines das gesamte Stück überwölbenden Oktavzugs an, so fällt auf, dass die Oberstimme der Takte 6-8 (siehe Beispiel 7) diese übergreifende melodische Entwicklung in gedrängter Form vorwegnimmt; nirgends in Les Ombres Errantes gibt es eine ähnliche Stelle. ${ }^{27}$ Ohnehin bildet die fragliche Phrase aufgrund ihrer zunächst subdominantischen Färbung des fast dissonanzfreien Satzes sowie der weitgehend abgeschwächten, auf das Kommende vorausweisenden harmonischen Zäsur in Takt 8 eine große Ausnahme - gerade ihre Schlichtheit, ja Schönheit hebt sie aus ihrer Umgebung heraus. Vorzitiert wird die mit $c^{3}$ ansetzende fallende Skalenbewegung bereits zuvor in der Unterstimme $\left(c^{2}-b^{1}-a s^{1}-g^{1}-f^{1}\right.$ in T. 6-7), und genau dort klingt sie - ebenfalls in der Unteroktave - im Anschluss imitierend nach ([ $\left[c^{2}\right]-b^{1}-a s^{1}-g^{1}-f^{1}-e s^{1}-d^{1}$ in T. 8-9). Mit ihren dissonanten Schärfungen bewirkt die Sequenzpassage der Takte 8-10 einen starken Binnenkontrast; gleichwohl zeichnet sie den eben durchmessenen Raum, im Diskant mit $b^{2}$ ansetzend, noch einmal in augmentierter Form nach. Da die erste Reprise in der Paralleltonart Es-Dur schließt, wird der übergreifende Urlinien-Oktavzug beim Wiederholungszeichen in der Terz geteilt. Es gehört zu den großen Vorzügen einer Schenker-Analyse, dass ihr Nachvollzug einen unmittelbaren Einfluss auf die Wahrnehmung hat, da sie den Blick für Stellen schärft, deren strukturelle Bedeutung sich erst im übergreifenden linearen Zusammenhang erschließt. Blickt man aus den beiden vorigen Perspektiven auf das Stück, so erscheinen die Takte 6-8 geradezu als ınebensächlich` (vgl. Beispiel 7).

\section{Aspekte satztechnischer Kombinatorik}

Ungeachtet der Schlichtheit des dreistimmigen Satzbildes ist die einleitende Phrase des Stückes reich an internen Beziehungen und weist zudem ausgeprägte Symmetrie-Merkmale auf (Beispiel 8).

Begreifen wir diese erste Phrase mit Schönberg als eine Art `Grundgestaltı und beschreiben ihre strukturellen Eigenschaften und motivischen Merkmale:

1. Von besonderer Bedeutung für die eröffnende Phrase ist das Intervall der verminderten Quarte: Es bildet den Rahmen des fallenden Tetrachords $e s^{1}-d^{1}-c^{1}-h$ in der Unterstimme (a) und verleiht als melodisches Spannungsintervall der viertönigen 'Knickfigur im Diskant (b) ihre charakteristische Prägung. ${ }^{28}$

2. Zwischen den Außenstimmen, die ein Gerüst aus Sexten und Dezimen bilden, findet zu Beginn ein fortwährender Austausch der Halbtonschritte $c-h$ und es- $d$ statt; auf diese Weise entsteht der Eindruck einer Vervielfältigung des fallenden Halbtonschritts - ein unmerklich swandernder Schattens?

27 Schenker führte für derartige Phänomene - es handelt sich hier im Grunde um eine »Wiederholung der Struktur des Ursatzes in einer späteren Schicht« - den Begriff des »Ursatzparallelismus« ein (vgl. Schwab-Felisch 2005, 350).

28 Zur Behandlung der verminderten (»kleinen«) Quart siehe Mattheson 1739, 290. Die melodische Kontur der Viertonfigur im Diskant entspricht dem Soggetto der cis-Moll-Fuge aus dem Wohltemperierten Klavier, Bd. 1. 

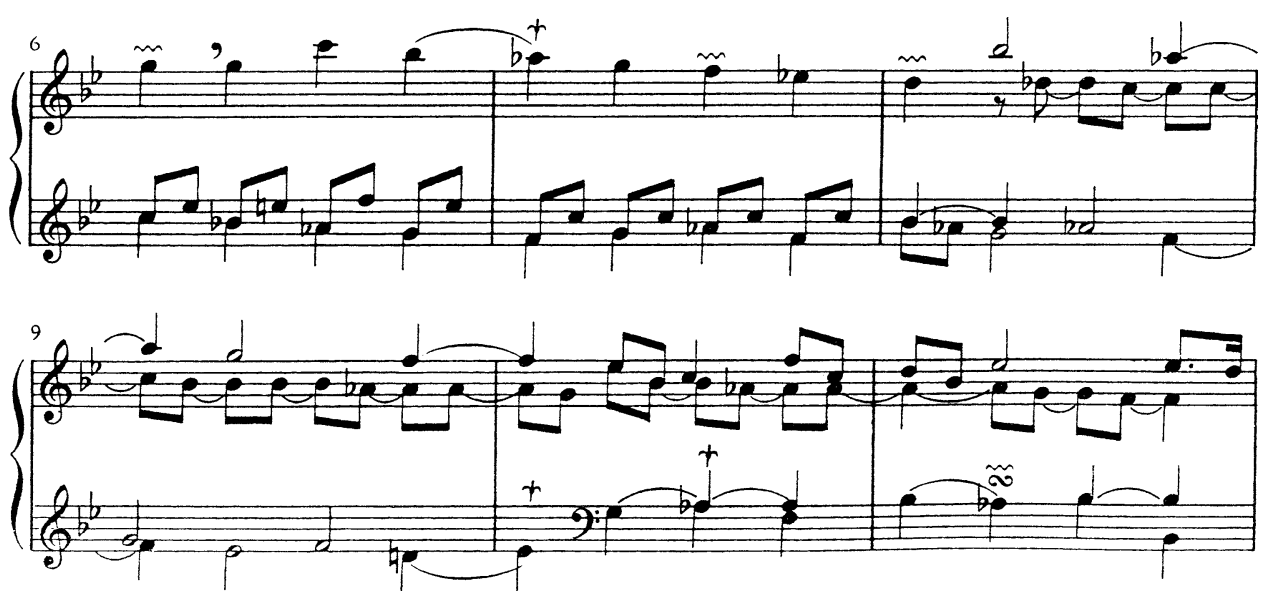

Beispiel 7: Les Ombres errantes, T. 6-11

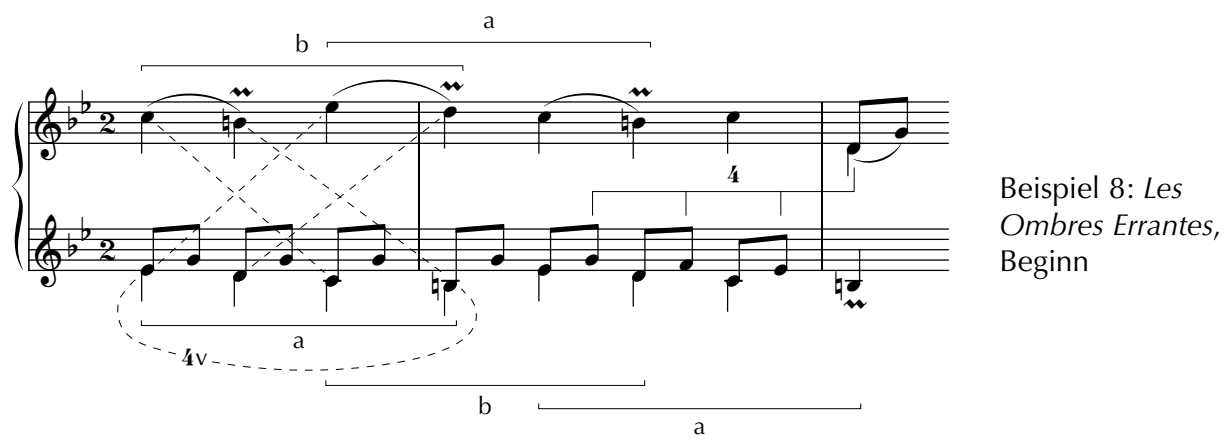

3. Die Bewegung der nachschlagenden Mittelstimme beschränkt sich zunächst auf ein Minimum, d. h. auf ein Pendeln zwischen $g^{1}$ und $f^{1}$; durch die Erweiterung hin zur Zäsur in Takt 2 entsteht ein zweites, in parallelen Terzen eingeführtes Tetrachord: $g^{1}-f^{1}-e s^{1}-d^{1}$. (Die Verunklarung der Stimmführung ist natürlich ein Erbe des sstyle brisér. $)^{29}$

Die Verarbeitung einzelner Merkmale der Grundgestalt (Beispiel 9) konzentriert sich auf den im Stück allgegenwärtigen Quartgang, der in beiden Bewegungsrichtungen und in verschiedenen Wertgrößen (auch synkopisch nachschlagend), in teilweise chromatisierter Gestalt (Takte 18-19) und als regelrechter passus duriusculus (Takte 16-17) erklingt. ${ }^{30}$

29 Nachdem sich Takt 2 endgültig sfestzupendeln` droht, erklingt dieser zweite (reine) Quartgang in Takt 3 dann als Oberstimme.

30 Als thematisches Intervalk ist die verminderte Quarte in Les Ombres Errantes von eher geringer Bedeutung. Immerhin jedoch kehrt der Quartgang in ursprünglicher Form - also mit dem verminderten Rahmenintervall - augmentiert zu Beginn der zweiten Reprise wieder (Takte 14-15: es-d-c-H), und im Penultima-Klang G-h-es (siehe Takt 27 in Entsprechung zu Takt 3) ist die verminderte Quarte ebenfalls enthalten. 
Grundgestalt

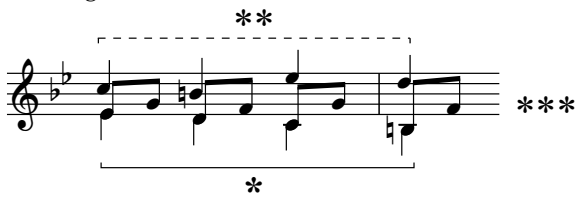

Verarbeitung
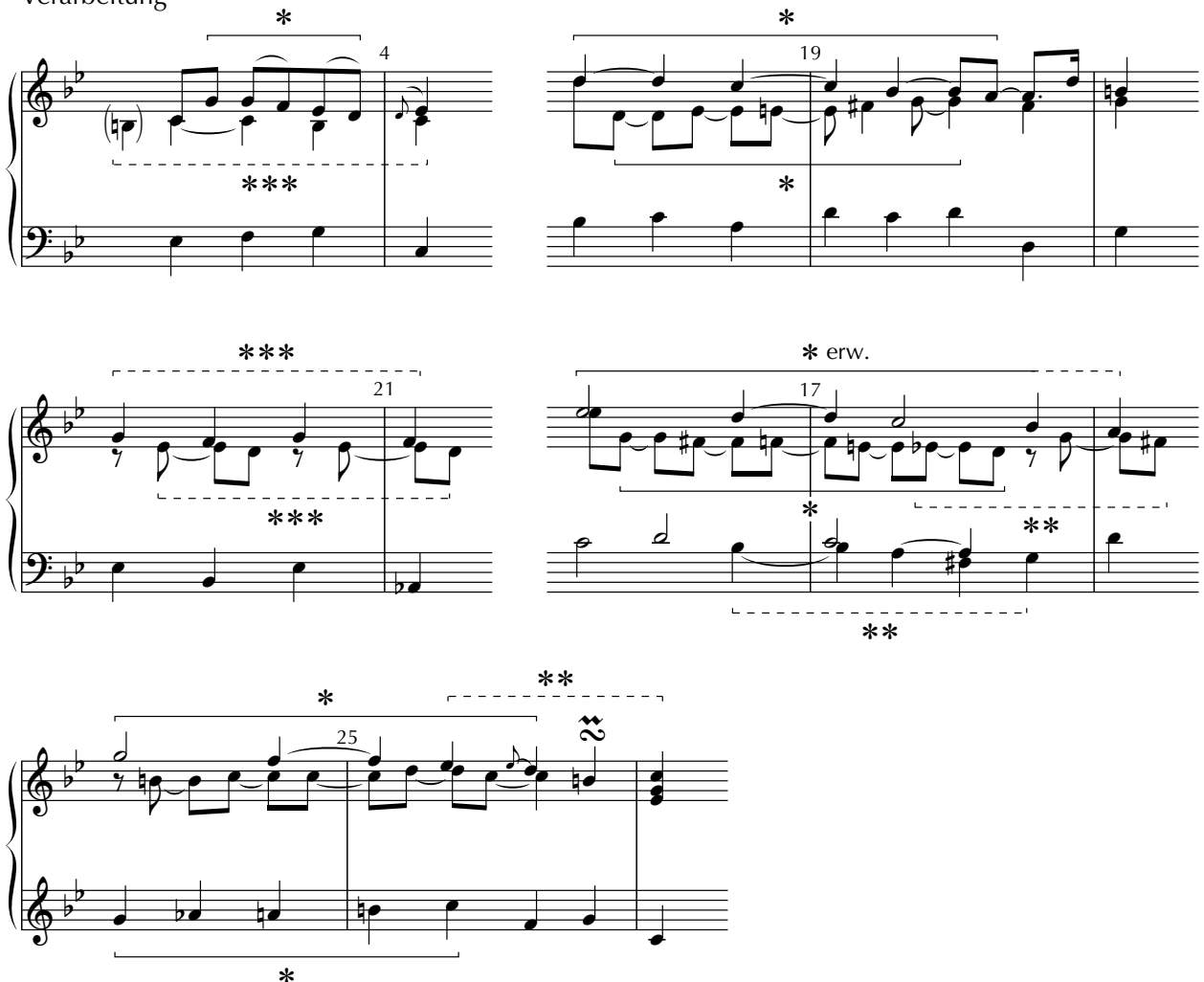

Beispiel 9: Les Ombres Errantes, Verarbeitung der Grundgestalt (* Quartgang; ** Knickfigur; *** Pendelfigur)

Aus der Simultankombination von recte- und inverso-Form entsteht in den Takten 18-19 ein sich schließender Fächer der Oberstimmen, kontrapunktiert von einer formelhaften Bassfigur, die wörtlich aus den Takten 10-12 übernommen ist. Die Fächer-Struktur wiederum findet sich in rhythmisch begradigter Form kurz vor Ende des Stückes im Außenstimmensatz der Takte 24-26 wieder. ${ }^{31}$ Aber auch die beiden anderen in der Grund-

31 Hier ist die 'Fächer-Idee` auf die Spitze getrieben, insofern das clusterartige Aufeinandertreffen der Töne $c^{2}, d^{2}$ und $e s^{2}$ (Takt $\left.25^{2}\right)$ den engsten Zusammenklang des gesamten Stückes bildet. 
gestalt enthaltenen melodischen Figuren, in der Grafik als 'Knickı- und als Pendelfigur bezeichnet, sind im Verlauf des Stückes allgegenwärtig, so die Knickfigur in den Takten 4-6 (als `Terz-Quart-Zickzackı), die Mittelstimmen-Pendelfigur beispielsweise in den Takten 21-22 und in den beiden Schlusstakten, die ihrerseits eine Art `conclusio ‘ darstellen, da sie sich darüber hinaus auch auf die Grundgestalt sowie die Takte 2-3 rückbeziehen. Je tiefer man in die Details eindringt, desto mehr verfestigt sich der Eindruck einer in hohem Maße stimmigen, wenngleich aus einem unscheinbaren, fast belanglosen Kern entwickelten Komposition (vgl. Beispiel 9).

Die aufgezeigten Verfahrensweisen beschränken sich nicht auf das Verkehren von Sätzen mit Hilfe des doppelten Kontrapunkts, wie es die zeitgenössische Kompositionslehre demonstriert. Es scheint Couperin in Les Ombres Errantes um ein Spiel mit Ähnlichkeiten und Differenzen, um locker-assoziative Bezugnahmen, motivische Vernetzungen scheinbar entlegener Punkte, um ein Moment verblüffender Kombinatorik zu gehen, das sowohl die Mikrostruktur der Grundgestalt als auch die Makrostruktur bestimmt. Um diesen `Beziehungszauber zu veranschaulichen, berücksichtigt die nachstehende Grafik (in Anlehnung an die in Abschnitt II entworfene Übersicht) neben den Entsprechungen von Einzelstimmen und Stimmpaaren auch die aufgezeigten motivischen Anspielungen zwischen den einzelnen Phrasen. Um die Grafik nicht zu überfrachten, sind nur die sinnfälligsten Bezüge skizziert.)

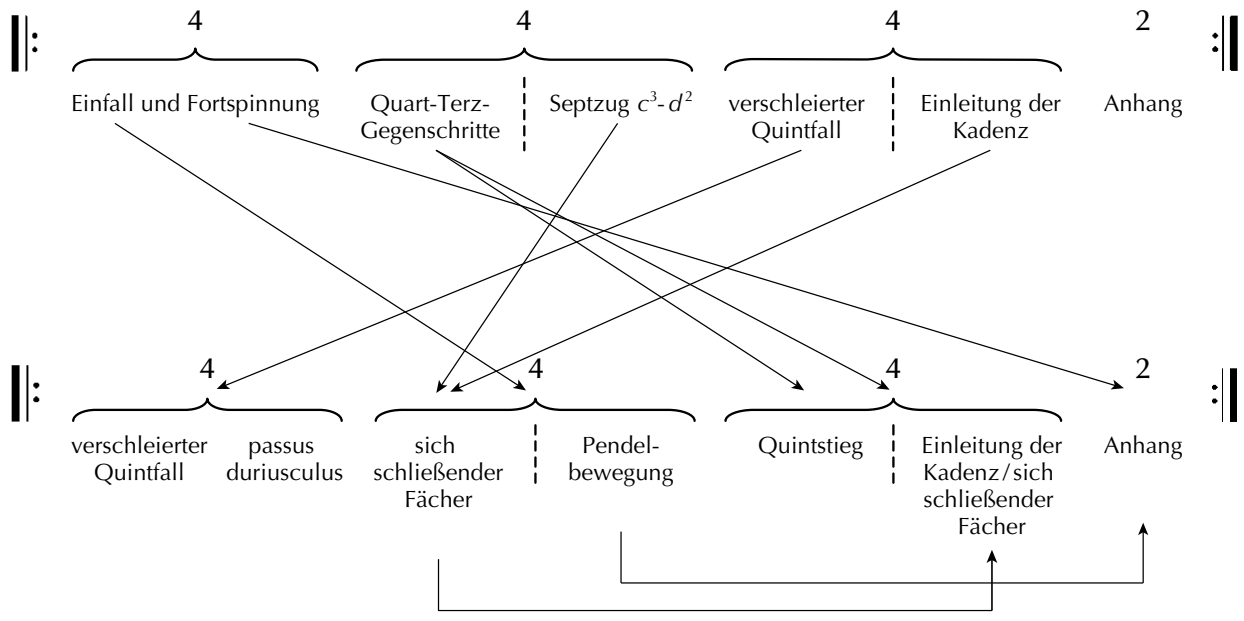

Abbildung 2: Les Ombres Errantes, >Beziehungszauber

Ein großer Teil der in Abschnitt IV zusammengetragenen Beobachtungen beruht auf einem Blickwinkel, den Arnold Schönberg in die Musiktheorie eingebracht hat. Insofern spiegeln die meisten Schlussfolgerungen Denkweisen wider, die etwa 200 Jahre jünger sind als das zur Diskussion stehende Stück. ${ }^{32}$ Ungeachtet des fast schwindelerregen-

32 Zur Geschichte des Grundgestalt-Begriffs siehe Beiche 1983/84. Einige exemplarische GrundgestaltAnalysen des Autors finden sich in Roth 2007. 
den Reichtums an Beziehungen, den die Analyse aufdecken konnte: Zweifel an dem Konzept, jedes motivische Detail in einem übergreifenden Zusammenhang aufgehen zu lassen, sind berechtigt. Dabei ließe sich diese Sichtweise noch zuspitzen: Nimmt man als Keimzelle des Stückes die eröffnende Parallelbewegung zweier Sextakkorde an (mit den zwei beteiligten kleinen Sekunden in den Außenstimmen), so entstehen durch Stimmtausch die Knickfigur sowie der verminderte Quartgang (auch in der Oberstimme), weiter (hin zu Takt 2) der reine Quartgang, der rasch erweitert (siehe den Septzug $c^{3}-d^{2}$ in T. 6-8) und im weiteren Verlauf auf mannigfaltige Weise verarbeitet wird. Und ließen sich dann nicht auch einige der im Stück auftretenden clusterartigen Zusammenklänge (z. B. es-f-g1-as ${ }^{1}$, Takt $14^{2}$ ) als vertikale Darstellung des zentralen Quartgangs verstehen?

\section{Fazit}

Les Ombres Errantes: Wie eingangs gezeigt wurde, liegt es nahe, die irregulären Vorhaltsbildungen innerhalb der sequenzierenden Abschnitte (T. 8-10 sowie T. 14-16) und das für den ıstyle brisé ‘ charakteristische `Verschwimmen` der Harmonie mit dem programmatischen Titel in Verbindung zu bringen. Freilich handelt es sich um zeittypische Satzmuster und Stilmittel, die bei Couperin und seinen Zeitgenossen auch in ganz anderen Zusammenhängen auftauchen: Ein hermeneutischer Zugang, der beim Rekurs auf das Satzbild stehenbliebe, griffe zu kurz. Welche darüber hinausgehenden Momente des Satzes aber assoziierte Couperin mit `Schattenwürfen`? Die besondere Beschaffenheit der `Grundgestalt‘? Ihre gleichsam unscharfe Symmetrie? Die minimale melodische Bewegung? Welche Bedeutung hat in diesem Zusammenhang der Gebrauch kontrastierender Lagen? Welche der ungewöhnliche dreizeitige Auftakt (sofern die Notation tatsächlich so zu verstehen ist)? Die heterogenen analytischen Befunde münden in Fragen: Werk und Titel bleiben rätselhaft. Es ist denkbar, hierin kein Defizit der Analyse, sondern eine Absicht des Komponisten zu erkennen. Insofern beruht die Faszination, die musikalische Kunstwerke auf uns ausüben, nicht zuletzt auf ihrer Vielschichtigkeit, die unterschiedlichste analytische Sichtweisen und damit Hörperspektiven zulässt. Entscheidend ist, was wir aus den Werken herauslesen.

\section{Literatur}

Bach, Carl Philipp Emanuel (1762), Versuch über die wahre Art, das Clavier zu spielen, Berlin, Reprint Kassel: Bärenreiter 1994.

Beaussant, Philippe (1980), François Couperin, Paris: Fayard.

Beiche, Michael (1983/84), Artikel Grundgestalt, in: HmT, 11. Auslieferung.

Couperin, François (1717), L'Art de toucher le clavecin, Paris, Reprint Wiesbaden: Breitkopf 1933.

Dahlhaus, Carl (1984), Die Musiktheorie im 18. und 19. Jahrhundert, Teil I: Grundzüge einer Systematik (= Geschichte der Musiktheorie 10), Darmstadt: Wissenschaftliche Buchgesellschaft. 
Edler, Arnfried (1997), Gattungen der Musik für Tasteninstrumente, Teil 1: Von den Anfängen bis 1750 (= Handbuch der musikalischen Gattungen 7,1), Laaber: Laaber.

Ebyl, Martin (2005), »Tonale Musik als vernetztes Stückwerk. Ein Merkmalkatalog der harmonischen Tonalität«, in: Zwischen Komposition und Hermeneutik. Festschrift für Hartmut Fladt, hg. von Ariane Jeßulat, Andreas Ickstadt und Martin Ullrich, Würzburg: Königshausen und Neumann, 54-66.

Fladt, Hartmut (2005), »Modell und Topos im musiktheoretischen Diskurs - Systematiken/Anregungen", Musiktheorie 20/4, 343-369.

Froebe, Folker (2007), „Satzmodelle des `Contrapunto alla mente und ihre Bedeutung für den Stilwandel um 1600«, ZGMTH 4/1-2, 13-56.

Händel, Georg Friedrich (1978), Aufzeichnungen zur Kompositionslehre (= Hallische Händel-Ausgabe, Supplement Bd. 1), hg. von Alfred Mann, Kassel u. a.: Bärenreiter.

Jans, Markus (1997), »Zur Rezeption der alten Musik in Theorie und Komposition«, Basler Jahrbuch für historische Aufführungspraxis 21, 87-100.

Kühn, Clemens / John Leigh (Hg.) (2009), Systeme der Musiktheorie, Dresden: Sandstein.

Mattheson, Johann (1739), Der vollkommene Capellmeister, Hamburg, Reprint, 5. Aufl., Kassel: Bärenreiter 1991 (= Documenta Musicologica, Druckschriften-Faksimiles 5).

Miehling, Klaus (2003), Das Tempo in der Musik von Barock und Vorklassik, 3. Aufl., Wilhelmshaven: Noetzel.

Roth, Markus (2007), Der Gesang als Asyl. Analytische Studien zu Hanns Eislers ıHollywood-Liederbuch (= sinefonia 7), Hofheim: Wolke.

Schönberg, Arnold (1933/1947) »Brahms, der Fortschrittliche«, in: ders., Stil und Gedanke, Frankfurt a. M. 1992: Fischer.

Smyth, David (1999), „Schenker's Oktave Lines Reconsidered«, Journal of music theory 43/1, 101-133.

Schwab-Felisch, Oliver (2004), „Die Abreißbarkeit der Gerüste«, Musiktheorie 19/4, 349-353.

— (2005), »Zur Schichtenlehre Heinrich Schenkers», in: Musiktheorie (= Handbuch der systematischen Musikwissenschaft 2), hg. von Helga de la Motte-Haber und Oliver Schwab-Felisch, Laaber: Laaber, 337-376.

Schwab-Felisch, Oliver / Hans-Ulrich Fuß, »Editorial«, ZGMTH 4/1-2, 9-12. 